\title{
Posturoterapia Neurossensorial e Acidente Vascular Cerebral: estudo preliminar
}

\author{
Neurosensory Posturotherapy and stroke: preliminary study
Posturoterapia neurosensorial y accidente cerebrovascular: estudio preliminar

\author{
Giovana Simão Bianchi ${ }^{1}$, Vagner Pires de Campos Junior ${ }^{2}$, Felipe \\ Sczepanski ${ }^{3}$, Cláudia Roberta Brunnquell Sczepanski ${ }^{4}$
}

\begin{abstract}
1.Fisioterapeuta, Universidade Estadual do Norte do Paraná. Centro de Ciências da Saúde (CCS). Jacarezinho-PR, Brasil. ORCID: https://orcid.org/0000-0001-5835-7799

2.Fisioterapeuta, Universidade Estadual do Norte do Paraná (UENP). Centro de Ciências da Saúde (CCS). Jacarezinho-PR, Brasil. ORCID: https://orcid.org/0000-0001-8279-6302

3. Cirurgião Dentista, Professor da Universidade Estadual do Norte do Paraná. Centro de Ciências da Saúde (CCS). Jacarezinho-PR, Brasil. ORCID: https://orcid.org/0000-0001-8030-4693

4.Fisioterapeuta, Professor Adjunto da Universidade Estadual do Norte do Paraná. Centro de Ciências da Saúde (CCS). Jacarezinho-PR, Brasil. ORCID: https://orcid.org/0000-0003-0336-7599
\end{abstract}

\section{Resumo}

Objetivo. Verificar o efeito da técnica de Posturoterapia Neurossensorial (PNS) na função pulmonar e qualidade de vida de indivíduos com Acidente Vascular Cerebral (AVC). Método. Participaram do estudo 14 indivíduos com AVC, de ambos os sexos, idade de $72,14 \pm 13,55$ anos e escore do mini mental de $22,29 \pm 6,83$. Foram submetidos à avaliação da força muscular respiratória, pico de fluxo expiratório, mobilidade torácica, comprometimento de tronco e qualidade de vida, e, após quatro semanas de intervenção com a técnica de PNS, foram reavaliados. Para a comparação utilizou-se $o$ teste $t$ pareado $(p<0,05)$. Resultados. Observou-se, após a intervenção, aumento da pressão expiratória máxima $(41,42 \pm 12,77$ para $48,93 \pm 11,63) \mathrm{cmH}_{2} \mathrm{O}$, pico de fluxo $(256,8 \pm 96,63$ para $290,7 \pm 90,17) \mathrm{l} / \mathrm{min}$, qualidade de vida $(81,30 \pm 21,36$ para $90,07 \pm 27,53)$ e diminuição do comprometimento de tronco $(16,07 \pm 2,165$ para 16,64 $\pm 2,274)$. Conclusão. Os achados deste estudo sugerem que as técnicas de PNS se mostraram eficazes na melhora da função pulmonar, controle de tronco e qualidade de vida de indivíduos acometidos por AVC.

Unitermos. Acidente vascular cerebral, fisioterapia, respiração, tronco

\begin{abstract}
Objective. To verify the effect of the Sensorineural Posturotherapy (SNP) technique on lung function and quality of life in individuals with stroke. Method. The study included 14 individuals with stroke, of both sexes, aged $72.14 \pm 13.55$ years and a mini mental score of $22.29 \pm 6.83$. They were evaluated and reassessed for respiratory muscle strength, peak expiratory flow, chest mobility, trunk impairment and quality of life, and after four weeks of intervention with the SNP technique. For comparison, the paired t-test $(p<0.05)$ was used. Results. After the intervention, there was an increase in the maximum expiratory pressure (41.42 \pm 12.77 to $48.93 \pm 11.63) \mathrm{cmH} 2 \mathrm{O}$, peak flow $(256.8 \pm 96.63$ to $290.7 \pm 90.17) \mathrm{l} / \mathrm{min}$, quality of life $(81.30 \pm 21.36$ to $90.07 \pm 27.53)$ and decrease trunk involvement (16.07 \pm 2.165 to $16.64 \pm 2.274)$. Conclusion. The findings of this study suggest that SNP techniques have been shown to be effective in improving lung function, trunk control and quality of life in individuals affected by stroke.
\end{abstract}

Keywords. stroke, physical therapy, respiratory, trunk 


\section{Resumen}

Objetivo. Verificar el efecto de la técnica de posuroterapia neurosensorial (PNS) sobre la función pulmonar y la calidad de vida en individuos con accidente cerebrovascular. Método. El estudio incluyó 14 individuos con accidente cerebrovascular, ambos sexos, 72,14士13,55

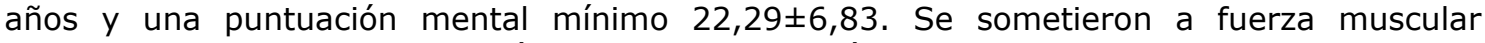
respiratoria, flujo espiratorio máximo, movilidad torácica, deterioro del tronco y calidad de vida, y después de cuatro semanas de intervención con la técnica PNS, fueron reevaluados. Para la comparación, se usó la prueba t pareada $(p<0,05)$. Resultados. Se observó, después de la intervención, un aumento en la presión espiratoria máxima $(41,42 \pm 12,77$ a $48,93 \pm 11,63) \mathrm{cmH} 20$, flujo máximo $(256,8 \pm 96,63$ a $290,7 \pm 90,17) \mathrm{l} / \mathrm{min}$, calidad de vida $(81,30 \pm 21,36$ a $90,07 \pm 27,53)$ and disminución del deterioro del tronco $(16,07 \pm 2,165$ a $16,64 \pm 2,274)$. Conclusión. a partir de este estudio, sugerimos que las técnicas de PNS serán efectivas en la función pulmonar, el control del tronco y la calidad de vida en personas afectadas por un accidente cerebrovascular.

Palabras clave. Accidente vascular cerebral, fisioterapia, respiración, tronco

Trabalho realizado na Universidade Estadual do Norte do Paraná - UENP. Centro de Ciências da Saúde CCS. Jacarezinho-PR, Brasil.

\section{INTRODUÇÃO}

O Acidente Vascular Cerebral (AVC) é definido pela Organização Mundial da Saúde (OMS) como uma perturbação focal da função cerebral, de origem vascular e início súbito, no qual os sintomas devem persistir por mais de 24 horas. Pode ser classificado como isquêmico, diagnosticado pelo bloqueio de um vaso sanguíneo responsável por alimentar o cérebro, e hemorrágico, quando há um sangramento ao redor ou dentro do cérebro ${ }^{1}$.

A isquemia e a hemorragia têm em comum uma manifestação clínica frequente, a hemiparesia ou hemiplegia, caracterizada por diminuição ou perda do controle motor do lado contralateral à lesão². A presença da espasticidade e hipotonia associadas à hemiparesia ou hemiplegia leva a um padrão de assimetria postural, com distribuição de peso 
menor sobre o lado afetado e transferência de peso corporal para o lado oposto, o que gera padrões anormais de movimento, fato este que pode prejudicar a mecânica ventilatória ${ }^{3}$.

O AVC é uma das principais causas de internações e mortalidade, levando, na maioria dos indivíduos, a algum tipo de deficiência, seja parcial ou completa ${ }^{4}$. Além disso, na anatomia, os músculos do tronco, bilateralmente, estão vinculados, sendo ao mesmo tempo agonistas e estabilizadores de uma ação. Por isso, a alteração da motricidade em um hemicorpo promove alterações musculares que comprometem o tronco como um todo, interferindo na biomecânica respiratória normal, comprometendo a função pulmonar ${ }^{5,6}$.

Sabe-se que a capacidade respiratória ideal necessita de uma postura de equilíbrio muscular perfeito. Porém, em indivíduos pós-AVC, o desequilíbrio muscular favorece o encurtamento da musculatura inspiratória, enquanto a musculatura abdominal se apresenta tensa e enfraquecida, sem atuação para abaixar a caixa torácica ${ }^{5,6}$.

A complacência torácica e pulmonar também se encontram diminuídas após o AVC, fato que provoca uma diminuição da capacidade pulmonar total e da capacidade vital, resultando em um distúrbio ventilatório restritivo na função pulmonar 6,7 . Com isso, é importante que 0 retreinamento e recuperação da atividade seletiva do tronco sejam partes integrantes do programa de reabilitação desses pacientes, facilitando o desempenho de atividades de vida 
diária e da marcha, diminuindo os riscos de quedas, além da melhora na função respiratória?.

As técnicas de Posturoterapia Neurossensorial (PNS) são uma forma de mobilização que pode conduzir a estes benefícios. Provém da técnica osteopática recoil, tendo sua característica maior na ligação entre os órgãos/sistemas com o cérebro ${ }^{8}$. Se a comunicação neural estiver obstruída e/ou comprometida, ela é utilizada, voltando a enviar informações ao Sistema Nervoso Central (SNC) e transportar informações dele para o Sistema Nervoso Periférico (SNP).

Quando realizada na região do tronco, passa a agir no sistema nervoso (propriocepção e analgesia), no tecido muscular (mantém características mecânicas), na pele (libera aderências), nas articulações (aumenta líquido sinovial, aumenta amplitude de movimento e nutre as cartilagens), na função respiratória (aumenta a amplitude de movimento das articulações que influenciam na ventilação) e na função digestiva (muda pressão intra-abdominal) ${ }^{9}$.

Assim, reabilitar esses indivíduos diminui a mortalidade e a morbidade, reduzindo os gastos tanto da família, quanto de saúde pública, além de aumentar a expectativa de vida desta população. Frente ao exposto, este estudo teve como objetivo verificar o efeito da intervenção com técnicas de PNS na função respiratória e qualidade de vida de indivíduos com AVC. 


\section{MÉTODO}

\section{Amostra}

Participaram do estudo 14 indivíduos acometidos por AVC, de ambos os sexos, com idade de 72,14 13,55 anos e Índice de Massa Corporal (IMC) de 27,03 $\pm 4,313 \mathrm{~kg} / \mathrm{m}^{2}$, considerados sobrepeso, e, escore do mini mental de

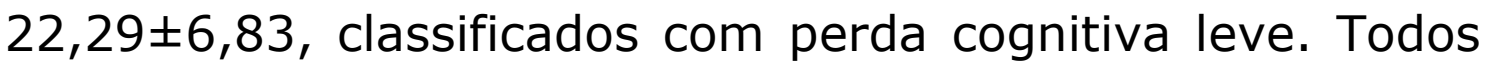
os participantes já realizavam fisioterapia convencional e foram convidados a participarem do estudo.

O estudo foi aprovado pelo Comitê de Ética em Pesquisa Institucional, sob o número $2.431 .810 \mathrm{e}$, todos os procedimentos realizados estavam de acordo com os padrões éticos de pesquisa envolvendo seres humanos e com a declaração de Helsinque de 1964 e suas posteriores alterações ou padrões éticos comparáveis.

A pesquisa tem Registro de Ensaio Clínico validado pelos critérios estabelecidos pela Organização Mundial de Saúde (OMS) e pelo ICMJE (Registro Brasileiro de Ensaios Clínicos - REBEC) com número de registro RBR-99j3h4.

Foram incluídos no estudo os indivíduos acometidos por AVC, hemodinamicamente estáveis e que assinaram o consentimento informado para a realização do estudo, Termo de Consentimento Livre e Esclarecido, permitindo a utilização dos dados coletados na pesquisa. Foram excluídos os indivíduos que não possuíam liberação médica para a prática da atividade, indisciplinados ou pouco colaborativos. 


\section{Procedimento}

Os participantes foram submetidos à avaliação, antes do tratamento proposto e, reavaliação, após quatro semanas de intervenção.

Foram coletados dados de idade, massa corporal e altura, para cálculo do Índice de Massa Corporal (IMC), pressão arterial sistêmica (esfigmomanômetro Aneroide Solidor $\left.{ }^{\circledR}\right)$, frequência cardíaca e saturação periférica (oxímetro portátil $50 \mathrm{DL}$ ).

A avaliação da força muscular respiratória foi realizada por meio da medição da pressão inspiratória máxima (PImáx) e pressão expiratória máxima (PEmáx) com o manovacuômetro analógico da marca WIKA®/Brasil, com intervalo operacional de $240 \mathrm{cmH}_{2} \mathrm{O}^{10}$. Os valores preditos foram calculados segundo equações propostas por Neder et al. ${ }^{11}$.

Para o pico de fluxo expiratório (PFE), caracterizado pela geração de fluxo máximo durante uma expiração forçada, executada partindo da CPT, utilizou-se o Peak Flow Meter (Vitalograph $®)^{12}$.

$\mathrm{Na}$ cirtometria tóraco abdominal são medidas as circunferências do tórax e abdômen no decorrer dos movimentos respiratórios. Para realizar a medição são utilizados três pontos como referência, sendo eles a prega axilar, processo xifoide e linha umbilical, demarcados com lápis dermatológico. Foi utilizada fita métrica escalonada em centímetros e as medidas anotadas foram durante a inspiração lenta máxima (CPT) e durante a expiração até o 
volume residual (VR). Após a realização das duas medidas foi feita a diferença entre elas ${ }^{13}$.

A Escala de Comprometimento do Tronco (ECT - Trunk Impairment Scale, TIS, no original) foi desenvolvida para mensurar os aspectos quantitativos do comprometimento do tronco do paciente hemiplégico ou hemiparético pós-AVC. A escala permite quantificar o comprometimento do tronco em sete itens. Dois itens (força muscular abdominal e verticalidade) foram originalmente baseados na SIAS (Stroke Impairment Assesment Set). Os outros cinco itens foram desenvolvidos para a ECT, consistindo na percepção de verticalidade do tronco, força de rotação dos músculos do tronco para os lados afetado e não-afetado, além das reações de endireitamento de ambos os lados. O escore para cada item varia de 0 a 3 e o melhor resultado corresponde à pontuação total de $21^{14}$.

A Escala de Qualidade de Vida Específica para AVE (EQVE-AVE) possui 49 itens em 12 domínios (energia, papel familiar, linguagem, mobilidade, humor, personalidade, autocuidado, papel social, raciocínio, função de membro superior, visão e trabalho/produtividade) e para cada item existem escores de até 5 pontos. O instrumento é de simples aplicação e compreensão dos itens, bem como na interpretação dos dados obtidos. Por ser uma escala específica, mostra-se válida para acessar alterações significativas da qualidade de vida dentro das dimensões dos sintomas observados, sendo uma escala direcionada às particularidades e domínios mais afetados nos pacientes 
após AVC. A pontuação consiste em: (1) Ajuda total: Não pude fazer de modo algum - Concordo inteiramente; (2) Muita ajuda: Muita dificuldade - Concordo mais ou menos; (3) Alguma ajuda: Alguma dificuldade - Nem concordo nem discordo; (4) Um pouco de ajuda: Um pouco de dificuldade - Discordo mais ou menos; e (5) Nenhuma ajuda necessária: Nenhuma dificuldade mesmo - Discordo inteiramente ${ }^{15}$.

A intervenção ocorreu uma vez por semana, durante quatro semanas, totalizando quatro sessões, de acordo com as orientações do método PNS, por meio das técnicas de saturação do nervo frênico, nervo das vértebras, nervo das costelas, nervo toracodorsal e nervo do dorso da escápula.

\section{Análise Estatística}

Os dados foram analisados no software GraphPadPrism 5.0 (GraphPad Software Inc., San Diego, CA, USA). Para avaliar a normalidade dos dados foi utilizado o teste de Shapiro-Wilk. Para a comparação das médias pré- e pósintervenção utilizou-se o teste t pareado $(p<0,05)$.

\section{RESULTADOS}

Na tabela 1 estão descritos os valores pré- e pósintervenção, descritos em média \pm desvio padrão.

Observamos que os valores de PEmáx, PFE, escores de ECT e EQVE-AVE mostraram aumento após a intervenção com a técnica de PNS, indicando que os indivíduos tiveram melhora da força muscular expiratória, fluxo expiratório, controle de tronco e qualidade de vida. 
Ao calcularmos o valor percentual do predito para as pressões respiratórias, verificamos que os indivíduos apresentaram, pré-intervenção, valores de PImáx e PEmáx de $63 \%$ e $39 \%$ do predito, respectivamente, indicando diminuição da força muscular respiratória.

Tabela 1. Comparação entre os valores pré e pós-intervenção com a técnica PNS.

\begin{tabular}{lcc}
\hline Variáveis & Pré-Intervenção & Pós-Intervenção \\
\hline PImáx & $50,00 \pm 14,81$ & $54,29 \pm 11,58$ \\
PEmáx & $41,42 \pm 12,77$ & $48,93 \pm 11,63^{*}$ \\
PFE & $256,8 \pm 96,63$ & $290,7 \pm 90,17^{*}$ \\
CA & $92,14 \pm 9,088$ & $92,00 \pm 8,124$ \\
CX & $94,43 \pm 8,102$ & $94,79 \pm 7,526$ \\
CB & $98,71 \pm 10,61$ & $98,43 \pm 9,874$ \\
CU & $102,3 \pm 10,19$ & $102,6 \pm 10,07$ \\
ECT & $16,07 \pm 2,165$ & $16,64 \pm 2,274 *$ \\
EQVE-AVE & $81,30 \pm 21,36$ & $90,07 \pm 27,53^{*}$
\end{tabular}

PImáx: Pressão Inspiratória Máxima; PEmáx: Pressão Expiratória Máxima; PFE: Pico de Fluxo Expiratório; CA: Cirtometria Axilar; CX: Cirtometria Xifoide; CB: Cirtometria Basilar; CU: Cirtometria Umbilical; ECT: Escala de Comprometimento de Tronco; EQVE-AVE: Escala de Qualidade de Vida Específica para AVE, $* p<0,01$.

\section{DISCUSSÃO}

No presente estudo, verificamos o efeito da intervenção com técnicas de PNS sobre a função respiratória e qualidade de vida de indivíduos com AVC.

Indivíduos com sequela neurológica estão predispostos a apresentar alteração na biomecânica respiratória, especialmente, os com alteração do tônus da musculatura torácica e/ou abdominal. As propriedades da caixa torácica 
modificam a ação dos músculos e o abdômen influencia a ventilação por meio da transmissão da pressão do conteúdo abdominal até o tórax, fato que pode interferir na biomecânica respiratória normal, gerando comprometimento da função pulmonar ${ }^{16}$.

Pacientes com sequelas de AVC podem apresentar comprometimento da mecânica respiratória, tendo suas pressões respiratórias máximas diminuídas, o que pode causar prejuízo na ventilação pulmonar, causando complicações como diminuição da ventilação pulmonar ${ }^{17}$.

Pesquisadores observaram que os valores para PImáx e PEmáx eram menores que o predito em indivíduos acometidos pelo AVC, e justificou o fato à disfunção diafragmática e abdominal ${ }^{18}$. Estes achados corroboram com nosso estudo que também revelou valores menores do predito para a população avaliada.

Um grupo de pacientes com AVC apresentou um aumento das pressões respiratórias máximas em cinco dias de treinamento de força muscular respiratória com ThresholdPEP ${ }^{19}$. Os resultados obtidos demonstram que os pacientes submetidos ao treinamento muscular inspiratório e expiratório apresentaram um aumento da PImáx e PEmáx em relação ao grupo controle. Em nosso estudo, houve aumento apenas da PEmáx, fato que pode ser justificado pela menor porcentagem do predito obtido na avaliação préintervenção.

Estudos anteriores mostraram correlação entre a PEmáx e o PFE com controle de tronco. Diante disso, 
inferiram que não só o tônus flácido interfere na mecânica respiratória, mas também a hipertonia e a fraqueza muscular persistente dos músculos do tronco exercem influência negativa na força muscular expiratória ${ }^{20}$. Assim, com os achados do nosso estudo, podemos sugerir que a melhora no controle de tronco com a técnica de PNS, pode aumentar tanto a força muscular expiratória quanto o pico de fluxo expiratório.

Em um estudo com 33 indivíduos acometidos por AVC, constatou-se que 16 horas de exercícios para melhora da função do tronco proporcionam maior equilíbrio, mobilidade e controle postural, sendo indispensáveis para reabilitação ${ }^{21}$. Ainda, a Facilitação Neuromuscular Proprioceptiva, usando as técnicas de reversão de estabilizações, reversão dinâmica e combinações de isotônicas podem promover fortalecimento muscular e efeitos importantes na estabilização do tronco em indivíduos com hemiparesia à esquerda decorrentes de $\mathrm{AVC}^{22}$. Desta forma, técnicas que objetivem a melhora da função do tronco poderiam, além de promover tais benefícios, contribuir para a melhora da função respiratória, como observado em nosso estudo.

O criador da PNS, salienta que o sistema tônico-postural (STP) é regulado pelo sistema nervoso central (SNC) e alimentado pela integração das entradas sensoriais. Para ocorrer essa comunicação, é de suma importância que as vias de comunicação estejam livres. É a rede nervosa que irá transportar as informações recolhidas das entradas sensoriais ao SNC e essa mesma rede transportará a 
resposta proveniente do SNC para o sistema de saída, ou seja, para o SNP23.

Se a comunicação neural estiver obstruída e/ou comprometida, tanto para levar informações ao SNC, quanto para transportar informações dele para o SNP, poderia haver inúmeras consequências, como limitação do movimento, sintomatologia persistente e manutenção da posição viciosa estática ${ }^{23,24}$. Portanto, a técnica utilizada, chamada saturações (regulação do tônus através de compressão e retirada brusca do nervo), fez com que houvesse a liberação das vias de comunicação, ou seja, os nervos somáticos, neurovegetativos e cranianos, promovendo alteração na função do tronco, tal qual ocorreu em nosso estudo.

A qualidade de vida relacionada à saúde específica de pessoas acometidas por AVC também se encontra diminuída e está associada a capacidade cognitiva. Maiores escores no Mini Exame do Estado Mental estiveram associados a melhores escores de qualidade de vida ${ }^{25}$. Assim, em nosso estudo, para fins de padronização, incluímos apenas indivíduos classificados com perda cognitiva leve, de acordo com o Mini Mental e observamos melhora na qualidade de vida após a intervenção.

\section{CONCLUSÃO}

Os achados deste estudo sugerem, pela primeira vez, que as técnicas de PNS se mostraram eficazes na melhora da força muscular e pico de fluxo expiratório, assim como no 
controle de tronco e qualidade de vida de indivíduos acometidos por AVC.

Assim, os resultados deste estudo preliminar justificam pesquisas futuras, com um maior número de pacientes acometidos por AVC, realizando a intervenção por um tempo maior e utilizando o método de PNS na íntegra, uma vez que o ponto forte do estudo foi a melhora significativa com a utilização da técnica apenas nos nervos da região do tronco.

\section{REFERÊNCIAS}

1.Kabuki MT, SÁ TS. Os efeitos da hidroterapia na hipertensão arterial e frequência cardíaca em pacientes com AVC. Rev Neurocienc 2007;15:131-4. https://doi.org/10.34024/rnc.2007.v15.10292

2. Makiyama TY, Battisttella LR, Litvoc J, Martins LC. Estudo sobre a qualidade de vida de pacientes hemiplégicos por Acidente Vascular Cerebral e de seus cuidadores. Rev Acta Fisiatr 2004;11:106-9. https://doi.org/10.5935/0104-7795.20040004

3. Mafalda L, Santos PH, Carrilho LO. Perfil respiratório de pacientes acometidos por Acidente Vascular Encefálico. Rev Saúde Integrada 2014;7:153-72. https://silo.tips/download/perfil-respiratorio-depacientes-acometidos-por-acidente-vascular-encefalico

4. Feigin VL, Abajobir AA, Abate KH, Abd-Allah F, Abdulle AM, Abera SF, et al. Global, regional, and national burden of neurological disorders during 1990-2015: a systematic analysis for the Global Burden of Disease Study 2015. Lancet Neurol 2017;16:877-97. https://dx.doi.org/10.1016/S1474-4422(17)30299-5

5.Zaleski TDP, Camera FD, Wisniewski E, Wisniewski MSW. Avaliação da força muscular respiratória e função pulmonar em indivíduos com Acidente Vascular Cerebral. Rev Perspectiva 2018;42:15-22. http://www.uricer.edu.br/site/pdfs/perspectiva/1005 655.pdf

6.Pompeu SMAA, Pompeu JE, Rosa M, Silva MR. Correlação entre função motora, equilíbrio e força respiratória pós Acidente Vascular Cerebral. Rev Neurocienc 2011;19:614-20.

https://doi.org/10.34024/rnc.2011.v19.8324

7.Ferla FL, Grav M, Perico E. Fisioterapia no tratamento do controle de tronco e equilíbrio de pacientes pós AVC. Rev Neurocienc 2015;23:211-7. https://doi.org/10.34024/rnc.2015.v23.8028

8.Torsten L, Dobler TB (ed.). Techniki osteopatyczne Tom 3. Polônia: Urban \& Partner; 2011.

9. Hengeveld E, Banks K. Maitland's Vertebral Manipulation E-Book: Management of Neuromusculoskeletal Disorders. 8th. Amsterdan: Elsevier Health Sciences, 2013; 472p. 
https://books.google.com.br/books/about/Maitland s Vertebral Mani pulation E Book.html?id=Gd6BAAAAQBAJ\&redir esc $=y$

10.Morais CS, Eickhoff CD, Pretto LM, Berlezi EM, Winkelmann ER. Avaliação da força muscular respiratória em indivíduos com Diabetes Mellitus tipo 2. Rev Contexto Saúde 2011;10:169-78. https://doi.org/10.21527/2176-7114.2011.20.169-178

11.Neder JA, Andreoni S, Lerario MC, Nery LE. Reference values for lung function tests. II. Maximal respiratory pressures and voluntary ventilation. Braz J Med Biol Res 1999;32:719-27. https://doi.org/10.1590/S0100-879X1999000600007

12. Nascimento MA, Santos GC, Freire RF. Avaliação do PeakFlow antes e após exercícios. Cad Grad 2015;2:11-20.

https://periodicos.set.edu.br/index.php/fitsbiosaude/article/view/183 $\underline{2}$

13.Caldeira VS, Starling CCD, Britto RR, Martins JA, Sampaio RF, Parreira VF. Precisão e acurácia da cirtometria em adultos saudáveis. J Bras Pneumol 2007;33:519-26.

http://dx.doi.org/10.1590/S180637132007000500006

14.Lima NMFV, Rodrigues SY, Fillipo TM, Oliveira R, Oberg TD, Cacho EWA. Versão brasileira da escala de comprometimento do tronco: um estudo de validade em sujeitos pós-acidente vascular encefálico. Fisioter Pesqu 2008;15:248-53. https://doi.org/10.1590/S180929502008000300006

15.Williams LS, Weinberger M, Harris LE, Clark DO, Biller J. Development of a stroke-specific quality of life scale. Stroke 1999;30:1362-9. http://dx.doi. org/10.1161/01.STR.30.7.1362

16.Cury JL, Pinheiro AR, Brunetto AF. Modificações da dinâmica respiratória em indivíduos com hemiparesia pós-acidente vascular encefálico. Assobrafir Cienc 2009;vol inaugural:5568. http://dx.doi.org/10.47066/2177-9333/ac.4185

17.Silva SM, Corrêa JCF, Silva FS, Sampaio LMM, Corrêa FI. Comparação da força muscular respiratória entre idosos após Acidente Vascular Cerebral. Acta Fisiatr 2013;20:20-3. https://doi.org/10.5935/0104-7795.20130004

18. Meneghetti CHZ, Figueiredo VE, Guedes CAV, Batistela ACT. Avaliação da Força Muscular Respiratória em Indivíduos Acometidos por Acidente Vascular Cerebral. Rev Neurocienc 2011;19:56-60. https://doi.org/10.34024/rnc.2011.v19.8398

19.Fernandes FE, Martins SRG, Bonvent JJ. Efeito do treinamento muscular respiratório por meio do manovacuômetro e do Threshold Pep em pacientes hemiparéticos hospitalizados. IFMBE Proceedings 2007;18:1199-202. https://doi.org/10.1007/978-3-540-74471-9 278 20.Jandt SR, Caballero RM, Junior LA, Dias AS. Correlation between trunk control, respiratory muscle strength and spirometry in patients with stroke: an observational study. Physiother Res Int 2011;16:21824. https://doi.org/10.1002/pri.495

21. Sayes W, Vereeck L, Truijen S, Lafosse C, Wuyts FP, Heyning PV. Randomized controlled trial of truncal exercises early after stroke to 
improve balance and mobility. Neurorehabil Neural Repair 2012;26:231-8. https://doi.org/10.1177/1545968311416822

22.Lacerda NN, Gomes EB, Pinheiro HA. Efeitos da Facilitação Neuromuscular Proprioceptiva na Estabilidade Postural e Risco de Quedas em Pacientes com Sequela de Acidente Vascular Encefálico: Estudo Piloto. Fisioter Pesq 2013;20:37-42.

https://doi.org/10.1590/S1809-29502013000100007

23. Villeneuve $P$. Dysfonctions neurales, algies posturales et neurostimulations manuelles. In: Weber B, Villeneuve P. Posturologie Clinique. Comprendre, évaluer, Soulager les Douleurs. Paris: Elsevier Masson, 2012, p.142-67. https://doi.org/10.1016/B978-2-29472130-4.00016-5

24.Veloso CF, Silveira AF, Garcia MV, Romero CAP. Osteopathic manipulation treatment on postural balance: a systematic review. MTP Rehab J 2016;14:352.

http://dx.doi.org/10.17784/mtprehabjournal.201 6.14.352

25.Canuto MAO, Nogueira LT, Araújo TME. Qualidade de vida relacionada à saúde de pessoas após acidente vascular cerebral, 2016. Acta Paul Enferm 2016;29:245-52. http://dx.doi.org/10.1590/1982$\underline{0194201600035}$ 